Bull. Mater. Sci., Vol. 2, Number 4, November 1980, pp. 265-270. (C) Printed in India.

\title{
Effect of an electric field on the oxidation of aluminium at room temperature
}

\author{
$K$ BHAVANI and $V \mathrm{~K}$ VAIDYAN \\ Department of Physics, University of Kerala, Trivandrum 695 581, India \\ MS received 30 November 1979 ; revised 16 October 1980
}

\begin{abstract}
Effect of an applied electric field on the oxidation rate of vacuum deposited aluminium thin films were studied at room temperature in the laboratory atmosphere. Above the critical field, a negative potential applied to the film enhanced the rate of oxidation, while a positive field retarded the rate. This suggests an oxidation mechanism involving cationic diffusion. The observed change in the rate of oxidation for an applied field agrees with the Mott-Cabrera theory.
\end{abstract}

Keywords. Aluminium oxidation; electric field; cationic diffusion; capacitive field.

\section{Introduction}

The mechanism of oxidation of metals cannot be fully understood from the study of kinetics alone, since different mechanisms can result in same rate law (Moore 1952; Ritchje and Hunt 1969). Hence to discover more about the nature of the rate determining step, it is necessary to carry out additional experiments. For aluminium in the temperature range between 50 and $400^{\circ} \mathrm{C}$, a detailed investigation of the mechanism of oxidation has been carried out to find out the influence of an applied electric field and also the effect of pressure changes of oxygen (Hunt and Ritchie 1972; Ritchie and Hunt 1969). At room temperature such a detailed. study is still lacking, though a few experiments were done (Boggio and Plumb 1966; Eley and Wilkinson 1960). In this paper, an attempt has been made to describe the reaction mechanism of the oxidation of aluminium at room temperature and the influence of an applied field across the growing oxide layer.

Field experiments have been carried out on several metals using different techniques. In early experiments, the electric field was applied across the oxide and an air gap, and little effect was observed (Uhlig and Brenner 1955; Cismaru and Cismaru 1961). In recent experiments, the field was applied between the metal film and an external electrode in contact with the oxide film. External electrodes used are in the form of wires (Shein et al 1961), porous films (Hunt and Ritchie 1970) or powders (Krishnamoorthy and Sircar 1968). In the present set-up, no external electrode is used. But, by keeping the metal film either at a positive or negative potential with respect to the ground, a capacitive field is developed across the metal oxide structure (Bhavani and Vaidyan 1981). 


\section{Experimental procedure}

Experiments were carried out on pure Indal aluminium thin films of thickness less than $500 \AA$. The metal film is deposited over glass slides by evaporation in vacuum from a tungsten basket. Thick aluminium filns are deposited at two ends of a glass slide for electrical contacts followed by a thin film at the central region. All evaporations were carried out at a pressure of the order of $10^{-5}$ torr. This aluminium was allowed to get oxidised in the laboratory atmosphere. The experiment was repeated at different times of the year when the humidity varied from 70 to $90 \%$.

The rate of oxidation can be monitored by measuring the resistance of the film. The change in resistance can be related to the oxide growth in the following manner. If it is assumed that the evaporated aluminium can be regarded as thin isotropic metal sheet which oxidises on one surface only, and obeys Ohm's law, then it follows that the film resistance $R$ and the cxide thickness $x$ are related by the equation (Hunt and Ritichie 1972),

$$
x=\frac{\phi \rho l}{\omega}\left(\frac{1}{R_{0}}-\frac{1}{R}\right),
$$

where $\phi$ is the thickness of oxide derived from unit thickness of metal, $\rho$ is the resistivity of the metal film, $l$ is the length of the film between the contacts, $\omega$ is the width of the film and $R_{0}$ is the resistance of the film prior to oxidation. In deriving this equation, it has been assumed that resistivity of the oxide is very much larger than that of the metal, a condition which is met by amorphous alumina and aluminium (Fisher and Giaever 1961). From equation (1), it is clear that the oxide thickness is proportional to $-1 / R$, so that the rate of change of resistance of the film is a measure of the rate of oxidation.

The metal film is kept either at a positive or negative potential with respect to the ground using a circuit arrangement shown in figure 1. A capacitive field is developed across the metal-metal oxide structure, as the metal is oxidised, and the magnitude and direction of the field depends on the potential applied to the film. For a potential of $200 \mathrm{~V}$, the voltage across the metal oxide is found to be $1 \cdot 2 \pm 0.1 \mathrm{~V}$. In a particular experiment the field is kept constant. At regular in tervals, the field is switched on and off; and the rate of oxidation is noted both

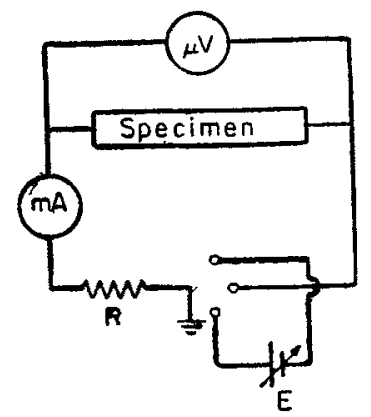

Figure 1. Experimental set-up. 
in the presence and absence of the field. Thus, a comparison for the rate of oxidation with and with sut an applied field is made for the same metal film. The experiment is repeated for different fisld strengths (corresponding to an applied voltage 30 to $200 \mathrm{~V}$ ) and also reversing the direstion of the field. To see that the results are not due to the effect of the current passing through the film the experiment is repeated for different values of the current ranging from $25 \mu \mathrm{A}$ to $3 \mathrm{~mA}$. It is confirmed that the effect is not due to the current flow.

\section{Results and discussion}

In general, a negative potential is found to have an acclerating effect (figure 2) and positive potential a retarding effect (figure 3 ) on the oxidation rate of

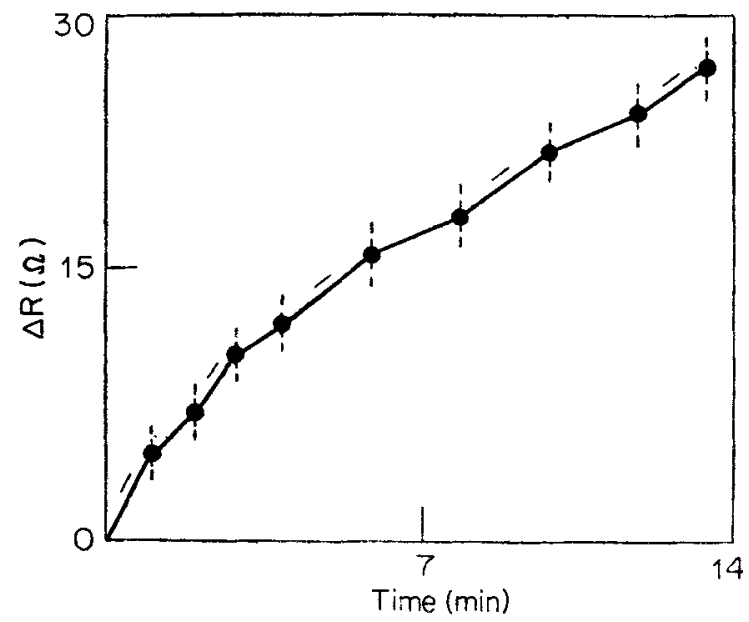

Figure 2. Comparison of oxidation rate with and without the application of a negative potential of $200 \mathrm{~V}$. Minus sign indicates the period of application of a negative potential.

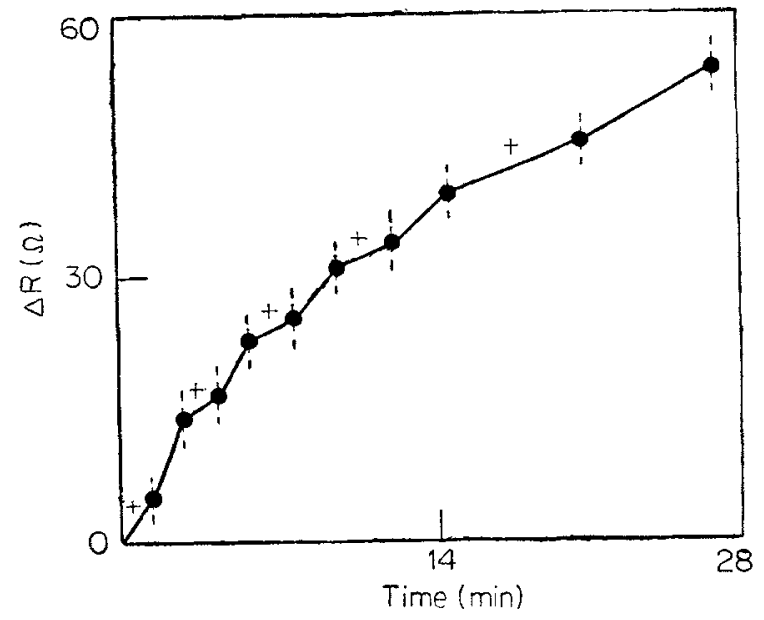

Figure 3. Comparison of oxidation rate with and without the application of a positive potential of $200 \mathrm{~V}$. Plus sign indicates the period of application of a positive potential. 
aluminium irrespective of the film thickness (the range of thickness was below $500 \AA$ ). When the metal film is at a negative potential, the direction of the field is such as to assist only the flow of positively charged cations. From the metalmetal oxide interface, and it can neither assist the diffusion of negatively charged electrons from the metal-metal oxide interface nor the flow of negatively charged oxygen jons through the oxygen-oxide interface. Thus, if the rate controlling factor of the oxidation mechanism is the flow of metal ions, an increase in the rate of oxidation can be expected when we apply a negative potential to the film. By the same argument we can say that the application of a positive potential can decrease the rate of diffusion of cations and hence the rate of oxidation. Thus, the observed results can be explained based on the fact that the rate of oxidation is controlled by the flow of cations from the metal to the oxide surface.

For low temperature region, Cabrera and Mott (1948-49) have postulated a theory based on field assisted ion transport. The Mott-Cabrera rate expression is

$$
r=d x / d t=N^{\prime} \Omega v \exp (-W / k T) \exp \left(q a^{\prime} F / k T\right)
$$

where $N^{\prime}$ is the number of atoms in kink sites at the oxide metal interface, $\Omega$ is) the volume of oxide formed permetal ion, $v$ is the frequency of vibration of the metal atoms, $W$ is the energy barrier to the movement of a metal ion from a kink site at the oxide metal interface into an interstitial position in the oxide, $q$ is the charge on a diffusing ion, $a^{\prime}$ is the distance from the bottom of the potential energy well of a kink site metal atom to the top of the energy barrier between the kink site an interestitial position in the oxide and $F$ is the electric field strength at the oxide-metal interface.

In the experimental situation, when we apply an external electric field of intensity $F_{0}$ across the oxide layer having a natural field $F_{0}$, the field across the oxide becomes)

$$
F=F_{0} \pm F_{e},
$$

the sign depends on the sense of the applied field. Equation (2) now becomes

$$
\begin{aligned}
r_{ \pm} & =N^{\prime} \Omega v \exp (-W / k T) \exp \left[q a^{\prime}\left(F_{0} \pm F_{e}\right) / k T\right] \\
& =r_{0} \exp \left( \pm q a^{\prime} F_{e} / k T\right),
\end{aligned}
$$

where $r_{0}$ is the rate of oxide growth with no external field. Thus, for the field which aids oxidation, we have

$$
\gamma_{+}=r_{+} / r_{0}=\exp \left(q a^{\prime} F_{\mathrm{e}} / k T\right)
$$

and for the field which opposes oxidation process,

$$
\gamma_{-}=r_{-} / r_{0}=\exp \left(-q a^{\prime} F_{e} / k T\right) \text {. }
$$

In our experiment, we have changed the field $F_{\theta}$ by changing the applied potential $E$, and for aluminium a negative potential aids the oxidation process while a positive potential reduces it. As the strength of the applied field, $F_{0}$ is proportional to $E$, and also from equations (5) and (6), we have

$$
\log \gamma \propto|E|
$$

where $\gamma=\gamma_{+}$or $1 / \gamma_{-}$for an applied potential $-E$ or $+E$, respectively. 
Table 1. Variation of $\gamma$ with electric field.

\begin{tabular}{cc}
\hline $\begin{array}{c}\text { Applied } \\
\text { potential } \\
|E|, V\end{array}$ & $\gamma^{*}$ \\
\hline 200 & $2.3 \pm 0.4$ \\
175 & $2.1 \pm 0.3$ \\
150 & $1.9 \pm 0.2$ \\
120 & $1.8 \pm 0.2$ \\
100 & $1.6 \pm 0.2$ \\
70 & $1.4 \pm 0.2$ \\
50 & $1.3 \pm 0.2$ \\
\hline
\end{tabular}

- Average value of $y_{+}$and $\left(y_{-}\right)^{-1}$ is taken as $\gamma_{\text {. }}$

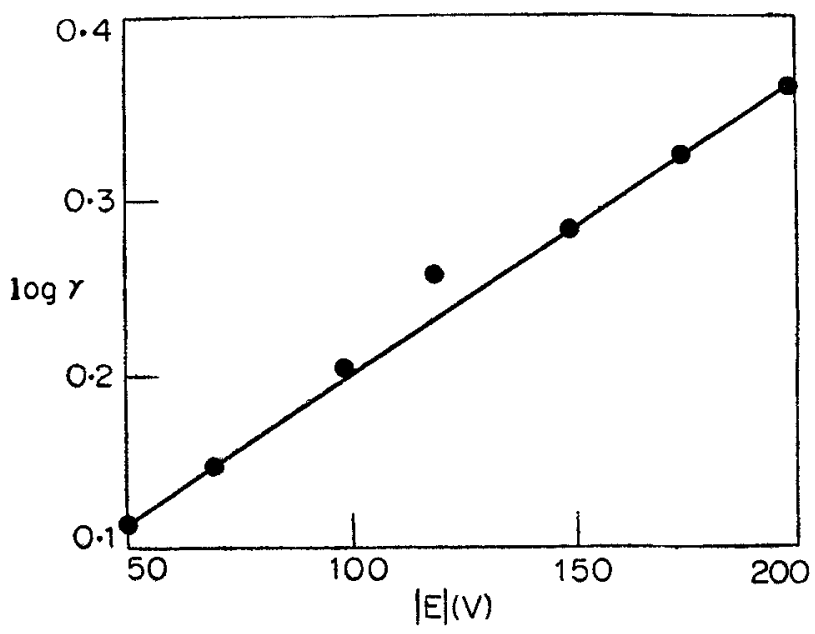

Figure 4.

From the oxidation-time graphs corresponding to a particular field strength, the ratios $\gamma_{+}$and $\left(\gamma_{-}\right)^{-1}$ are determined at different regions of the curves, and their mean value is taken as $\gamma$. The ratios thus determined at different field strengths corresponding to applied potentials in the range 50 to $200 \mathrm{~V}$ are given in table 1 . A graph is plotted with $\gamma$ vs. $|E|$ (figure 4). The straight line nature of the graph within the limits of experimental errors shows the validity of equation (7).

In the present experimental set-up, for fields corresponding to an applied potential less than $\pm 50 \mathrm{~V}$, no change in the rate of oxidation was observed. At this field strength, $y$ reaches a value about $1 \cdot 3 \pm 0 \cdot 2$, and such a small change in the ratio of rate factors is very difficult to detect experimentally. 


\section{Conclusion}

At room temperature, oxide growth on evaporated aluminium films has been found to proceed by metal transport. The variation of the magnitude of the change in rate of oxidation with the applied field is found to be in agreement with the predictions of the Mott-Cabrera theory.

\section{Acknowledgement}

One of the authors (KB) thanks the Council of Scientific and Industrial Research, New Delhi, for financial assistance.

\section{References}

Bhavani K and Vaidyan V K 1981 Oxid. Met. 15137

Boggio J E and Plumb R C 1966 J. Chem. Phys. 441081

Cabrera N and Mott N F 1948-49 Report Progr. Phys. 12163

Cismaru D and Cismaru G D 1961 Proc. I Int. Cong. of Metallic Corrosion (London) p. 194

Eley D D and Wilkinson P R 1960 Proc. R. Soc. (London) 254327

Fisher J C and Giaever I $1961 \mathrm{~J}$. Appl. Phys. 32172

Hunt G L and Ritchie I M 1970 Oxid. Met. 2361

Hunt G L and Ritchie I M 1972 J. Chem. Soc. Faraday Trans. I. 681413

Krishnamoorthy P K and Sircar S C 1968 Acta Metall. 161461

Moore W J 1952 Philos. Mag. 43688

Ritchie I M and Hunt G L 1969 Surf. Sci. 15524

Shein F, Le Bouncher B and Lacompe P 1961 CR Acad. Sci. (Paris) 2524157

Uhlig H H and Brenner A E 1955 Acta Metall. 3108 